\title{
A MODEL GENERATOR FOR THE PHYSICS LABORATORY.
}

\author{
By Henry Kempner, \\ Pratt Institute, Brooklyn, N. Y.
}

The model generator described in this article is used in the physics laboratory at Pratt Institute to familiarize the student with the principles of the electric generator; how it produces electrical energy, the nature of the E. M. F. produced and the construction of the machine.

The generator consists of the frame, field coils and drum armature of a standard Allis-Chalmers $2 \mathrm{H}$-P generator. On the armature are wound three coils easily distinguishable one from the other and each of which is brought out to a pair of collector rings. The collector rings and brushes are the standard parts of the generator. On the pulley end of the armature shaft, which projects about $3 \frac{1}{2}$ " from the generator frame, is mounted a handle and ratchet arrangement by means of which the armature can be snapped around by hand 15 degrees at a time.

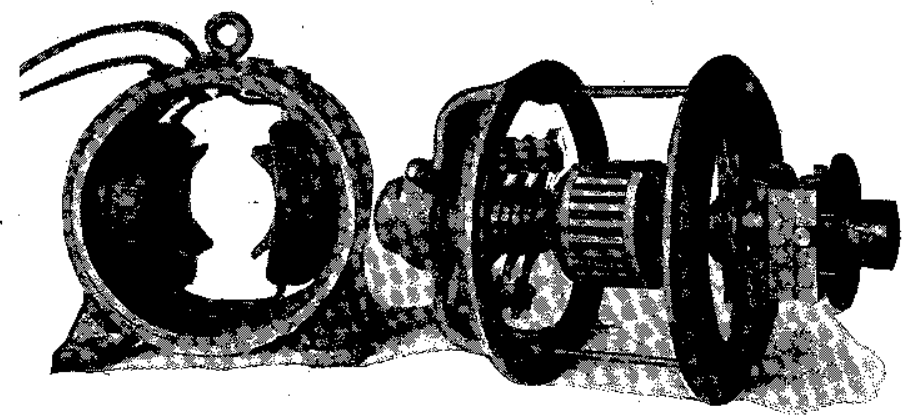

This generator is used in an experiment entitled, "Model generator: Instantaneous voltages induced in an armature," and folm lowing is a copy of the direction sheet covering the experiment taken from the students' laboratory manual.

Model Generator: Instantaneous Voltages in an Armature.

Apparatus: Model generator, $1.5-150$ volt D. C. Voltmeter, fuses, source of 110 volts pressure.

Method: Excite the fields of the generator. Connect the voltmeter to two brushes which are terminals of one of the armature coils. By means of the handle and ratchet snap the armature around 15 degrees and note the direction of the reading of the instrument. If it does not read in the proper direction reverse the voltmeter connections and repeat. Continue taking readings 
until they fall to zero, then reverse the voltmeter connections and continue until you have taken a total of at least 24 readings. Care should be taken in snapping the armature around to move the handle at about the same rate for each reading.

It is advisable to try out the apparatus by taking a preliminary set of data in order. that you may be sure just when to reverse the voltmeter connections.

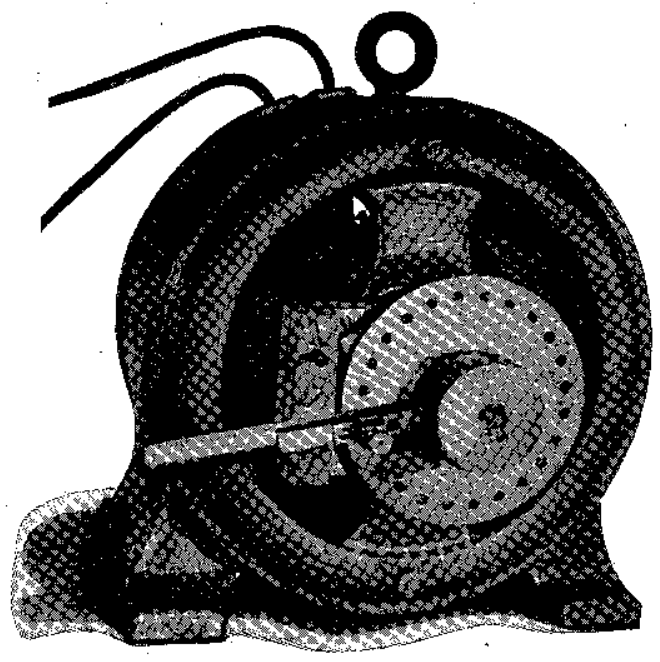

From the direction of the field flux and direction of rotation of the armature determine by use of the right-hand rule the direction of the induced $\mathrm{E} . \mathrm{M}$. F. If the direction of the current through the field coils is reversed what would be the effect on the induced E. M. F.? Reverse your field connections and check.

Data and Results: Plot a curve of the induced E. M. F. On the $\mathrm{X}$ axis plot the angle of the armature coil with the vertical. On the $\mathrm{Y}$ axis plot the value of the voltage read paying due attention to sign.

In red ink on the same paper plot the curve $\mathrm{Y}=\mathrm{E} \max \operatorname{Sin} \mathrm{X}$. Compare the two curves.

The experiment may be added to further by having the student remove the ratchet arrangement from the end of the armature shaft and connecting the armature to a motor. In this way the effect of the speed of the armature on the induced E. M. F., can be studied. By varying the strength of the field current and keeping the speed constant the effect of increased or decreased flux on the induced E. M. F. can be determined.

An interesting feature of the generator is the uniformity of 
results obtained from it. The two curves of the induced E. M. $F$. shown represent the best and the worst results obtained by average students in a class of 35 .

The machine has been in use for over a year during which period approximately 245 students have performed the experiment. During that time the only attention required by the apparatus has been the oiling of the shaft bearings. The machine having been originally built for commercial use should stand up easily under the use to which it is now being subjected for a long time.

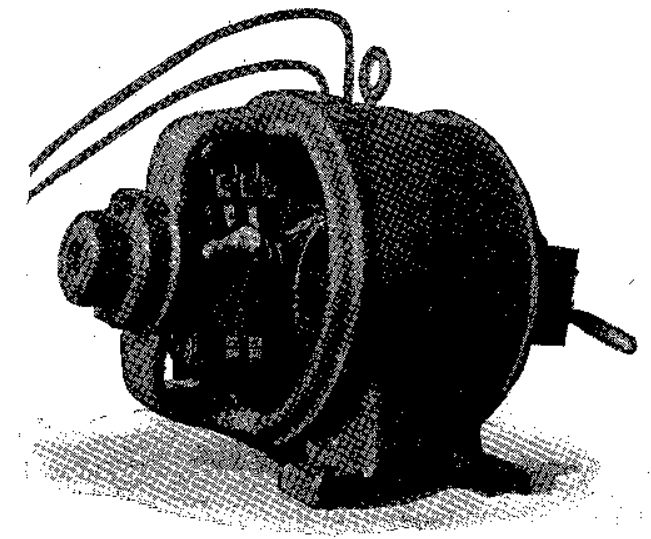

This model generator has particular value to the student of the Pratt Institute type, who must get a considerable amount of theoretical and practical training in a short time, because it is a commercial piece of apparatus. It looks like the other generators the student sees around the laboratory and about the city. It is like those he will see when he gets out into actual commercial life. He is getting his theory from an actual piece of apparatus and consequently that theory becomes associated with a mental picture of the apparatus. He sees how the generator is built to conform to the laws governing an induced E. M. F. and also subconsciously absorbs standards of good commercial design and workmanship. The reasons for certain details in the design and construction of the generator are in this way made clear to the student and the one tending to recall the other will help him to remember both the theory and the construction.

A movement to introduce commercial apparatus into the laboratories appears to be developing and it should tend considerably. toward lessening the impression that the school graduate is theoretical and not practical. Certainly such apparatus helps to make the laboratory exercises concrete problems. 